\title{
Non Gaussian Minkowski functionals and extrema counts for CMB maps
}

\author{
Dmitri Pogosyan ${ }^{1,2,3}$, Sandrine Codis ${ }^{2}$ and Christophe Pichon ${ }^{2}$ \\ ${ }^{1}$ Department of Physics, University of Alberta, 11322-89 Avenue, Edmonton, Alberta, T6G \\ 2G7, Canada \\ ${ }^{2}$ CNRS, UPMC, Institut d'astrophysique de Paris, 98 bis boulevard Arago, 75014, Paris, \\ France \\ ${ }^{3}$ CNRS, Institut Lagrange de Paris, 98 bis boulevard Arago, 75014, Paris, France
}

\begin{abstract}
In the conference presentation we have reviewed the theory of non-Gaussian geometrical measures for 3D Cosmic Web of the matter distribution in the Universe and 2D sky data, such as Cosmic Microwave Background (CMB) maps that was developed in a series of our papers. The theory leverages symmetry of isotropic statistics such as Minkowski functionals and extrema counts to develop post Gaussian expansion of the statistics in orthogonal polynomials of invariant descriptors of the field, its first and second derivatives. The application of the approach to $2 \mathrm{D}$ fields defined on a spherical sky was suggested, but never rigorously developed. In this paper we present such development treating the effects of the curvature and finiteness of the spherical space $S_{2}$ exactly, without relying on flat-sky approximation. We present Minkowski functionals, including Euler characteristic and extrema counts to the first non-Gaussian correction, suitable for weakly non-Gaussian fields on a sphere, of which CMB is the prime example.
\end{abstract}

Random fields are ubiquitous phenomena in physics appearing in areas ranging from turbulence to the landscape of string theories. In cosmology, the sky-maps of the polarized Cosmic Microwave Background (CMB) radiation - a focal topic of current research - is a prime example of such $2 \mathrm{D}$ random fields, specified on $S_{2}$ spherical space. Modern view of the cosmos, developed primarily through statistical analysis of these fields, points to a Universe that is statistically homogeneous and isotropic with a hierarchy of structures arising from small Gaussian fluctuations of quantum origin. While the Gaussian limit provides the fundamental starting point in the study of random fields, non-Gaussian features of the CMB fields are of great interest. Indeed, CMB inherits a high level of Gaussianity from initial fluctuations, but small non-Gaussian deviations may provide a unique window into the details of processes in the early Universe. The search for the best methods to analyze non-Gaussian random fields is ongoing.

In the paper Pogosyan, Gay \& Pichon (2009) the general invariant based formalism for computing topological and geometrical characteristics of non Gaussian fields was presented. The general formulae for the Euler characteristic to all orders has been derived, which encompasses the well known first correction Matsubara (2003) and which was later confirmed to the next order by Matsubara (2010). This work was followed by the detailed exposition of the theory in 2D and 3D flat (Cartesian) space in Pogosyan, Pichon \& Gay (2011) and Gay, Pichon \& Pogosyan (2012), and generalized to the 3D redshift space where isotropy is broken in Codis et al. (2013).

The goal of this paper is to extend these results to the fields defined on a finite curved spherical space $S_{2}$ without reliance on the flat field (small angle) approximation. While these proceedings were being prepared, similar work has been done for statistics of peaks in the Gaussian case within Marcos-Caballero et al. (2016). Here our focus is on nonGaussian corrections. We discuss how to compute exact Minkowski functionals for the 
excursion sets of a scalar field on a $S_{2}$ sphere to all orders in non-Gaussian expansion and provide an explicit expression for the Euler characteristic to the first order. Expressions for the total extrema counts to the first non-Gaussian order are also given, while analytical formulas for differential extrema counts to the same order will be published elsewhere due to their length. These results have a direct relevance to CMB data analysis.

\section{Joint distribution function of the field and its derivatives on $S_{2}$ sphere.}

The statistics of Minkowski functionals, including the Euler number, as well as extrema counts requires the knowledge of the one-point joint probability distribution function (JPDF) $P\left(x, x_{i}, x_{i j}\right)$ of the field $x$, its first, $x_{i}$, and second, $x_{i j}$, derivatives of the field $x$. Let us consider random field $x$ defined on a $2 \mathrm{D}$ sphere $S_{2}$ of radius $R$ represented as the expansion in spherical harmonics

$$
x(\theta, \phi)=\sum_{l=0}^{\infty} \sum_{m=-l}^{l} a_{l m} Y_{l m}(\theta, \phi)
$$

where for the Gaussian statistically homogeneous and isotropic field random coefficients $a_{l m}$ are uncorrelated with $m$-independent variances $C_{l}$ of each harmonic

$$
\left\langle a_{l m} a_{l^{\prime} m^{\prime}}^{*}\right\rangle=C_{l} \delta_{l l^{\prime}} \delta_{m m^{\prime}}
$$

The variance of the field is then given by

$$
\sigma^{2} \equiv\left\langle x^{2}\right\rangle=\frac{1}{4 \pi} \sum_{l} C_{l}(2 l+1)
$$

When considering derivatives in the curved space, we use covariant derivatives $x_{; \theta}, x_{; \phi}$, $x_{; \theta}^{; \theta}, x_{; \phi}^{; \phi}, x^{; \theta} ; \phi$ where it will be seen immediately that mixed version for the second derivatives is the most appropriate choice. The $2 \mathrm{D}$ rotation-invariant combinations of derivatives are

$$
q^{2}=x_{; \phi} x^{; \phi}+x_{; \theta} x^{; \theta}, J_{1}=\left(x_{; \theta}^{; \theta}+x_{; \phi}^{; \phi}\right)^{2}, J_{2}=\left(x_{; \theta}^{; \theta}-x_{; \phi}^{; \phi}\right)^{2}+4 x_{; \phi}^{; \theta} x_{; \theta}^{; \phi}
$$

where $J_{1}$ is linear in the field and $q^{2}$ and $J_{2}$ are quadratic, always positive, quantities. The derivatives are also random Gaussian variables, which variances are easily computed

$$
\begin{aligned}
\sigma_{1}^{2} & \equiv\left\langle q^{2}\right\rangle=\frac{1}{4 \pi R^{2}} \sum_{l} C_{l} l(l+1)(2 l+1) \\
\sigma_{2}^{2} & \equiv\left\langle J_{1}^{2}\right\rangle=\frac{1}{4 \pi R^{4}} \sum_{l} C_{l} l^{2}(l+1)^{2}(2 l+1) \\
\sigma_{2}^{\prime 2} & \equiv\left\langle J_{2}\right\rangle=\frac{1}{4 \pi R^{4}} \sum_{l} C_{l}(l-1) l(l+1)(l+2)(2 l+1)
\end{aligned}
$$

where the fundamental difference between a sphere and the 2D Cartesian space is in the fact that $\sigma_{2}^{\prime} \neq \sigma_{2}$. Among the cross-correlations the only non-zero one is between the the field and its Laplacian $\left\langle x\left(x_{; \theta}^{; \theta}+x_{; \phi}^{; \phi}\right)\right\rangle=-\sigma_{1}^{2}$.

From now on we rescale all random quantities by their variances, so that rescaled variables have $\left\langle x^{2}\right\rangle=\left\langle J_{1}^{2}\right\rangle=\left\langle q^{2}\right\rangle=\left\langle J_{2}\right\rangle=1$. Introducing $\zeta=\left(x+\gamma J_{1}\right) / \sqrt{1-\gamma^{2}}$ (where the spectral parameter $\gamma=-\left\langle x J_{1}\right\rangle=\sigma_{1}^{2} /\left(\sigma \sigma_{2}\right)$ ) leads to the following JPDF for 
the Gaussian 2D field

$$
G_{2 \mathrm{D}}=\frac{1}{2 \pi} \exp \left[-\frac{1}{2} \zeta^{2}-q^{2}-\frac{1}{2} J_{1}^{2}-J_{2}\right] .
$$

In Pogosyan, Gay \& Pichon (2009) we have observed that for non-Gaussian JPDF the invariant approach immediately suggests a Gram-Charlier expansion in terms of the orthogonal polynomials defined by the kernel $G_{2 \mathrm{D}}$. Since $\zeta, q^{2}, J_{1}$ and $J_{2}$ are uncorrelated variables in the Gaussian limit, the resulting expansion is

$$
\begin{aligned}
& P_{2 \mathrm{D}}\left(\zeta, q^{2}, J_{1}, J_{2}\right)=G_{2 \mathrm{D}}[1+ \\
& \left.\sum_{n=3}^{\infty} \sum_{i, j, k, l=0}^{i+2 j+k+2 l=n} \frac{(-1)^{j+l}}{i ! j ! k ! l !}\left\langle\zeta^{i} q^{2^{j}} J_{1}{ }^{k} J_{2}{ }^{l}\right\rangle_{\mathrm{GC}} H_{i}(\zeta) L_{j}\left(q^{2}\right) H_{k}\left(J_{1}\right) L_{l}\left(J_{2}\right)\right],
\end{aligned}
$$

where terms are sorted in the order of the field power $n$ and $\sum_{i, j, k, l=0}^{i+2 j+k+2 l=n}$ stands for summation over all combinations of non-negative $i, j, k, l$ such that $i+2 j+k+2 l$ adds to the order of the expansion term $n . H_{i}$ are (probabilists') Hermite and $L_{j}$ are Laguerre polynomials. The coefficients of expansion

$$
\left\langle\zeta^{i} q^{2^{j}} J_{1}^{k} J_{2}^{l}\right\rangle_{\mathrm{GC}}=\frac{j ! l !}{(-1)^{j+l}}\left\langle H_{i}(\zeta) L_{j}\left(q^{2}\right) H_{k}\left(J_{1}\right) L_{l}\left(J_{2}\right)\right\rangle .
$$

are related (and for the first non-Gaussian order $n=3$ are equal) to the moments of the field and its derivatives (see Gay, Pichon \& Pogosyan (2012) for details).

Up to now our considerations are practically identical to the theory in the Cartesian space, which facilitates using many of the Cartesian calculations. We stress again the only, but important difference being $\sigma_{2}^{\prime} \neq \sigma_{2}$. We shall see in the next sections how this difference plays out. Here we introduce the spectral parameter $\beta$ that describes this difference

$$
\beta \equiv 1-\frac{\sigma_{2}^{\prime 2}}{\sigma_{2}^{2}}=2 \frac{\sum_{l} C_{l} l(l+1)(2 l+1)}{\sum_{l} C_{l} l^{2}(l+1)^{2}(2 l+1)}
$$

Let us review the scales and parameters that the theory has. As in the flat space, we have two scales $R_{0}=\sigma / \sigma_{1}$ and $R_{*}=\sigma_{1} / \sigma_{2}$ and the spectral parameter $\gamma=R_{*} / R_{0}$ (which also describes correlation between the field and its second derivatives). On a sphere we have a third scale, the curvature radius $R$. The meaning of $\beta$ becomes clear if we notice that $\sigma_{2}^{2}-\sigma_{2}^{\prime 2}=2 \sigma_{1}^{2} / R^{2}$, thus $\beta=2 R_{*}^{2} / R^{2}$, i.e describes the ratio of the correlation scale $R_{*}$ to the curvature of the sphere. As with the $\gamma, \beta$ varies from 0 to 1 , with $\beta=0$ corresponding to the flat space limit. From Eq. (1.11) we find that $\beta=1$ is achieved when the field has only the monopole and the dipole in its spectral decomposition.

\section{Minkowski functionals on $S_{2}$ beyond the Gaussian limit}

There are three Minkowski functionals that are defined for the excursion set above threshold $\nu$ of a 2D field, namely the filling factor, $f_{V}(\nu)$, i.e the volume fraction occupied by the region above the threshold $\nu$, the length (per unit volume) of isofield contours, $\mathcal{L}(\nu)$ and Euler characteristic $\chi(\nu)$. Statistics of the first two do not depend on second derivatives of the field, and thus are identical on $S_{2}$ and the 2D Cartesian space. Here, for completeness, we reproduce the non-Gaussian expansions for these quantities from 
Gay, Pichon \& Pogosyan (2012)

$$
\begin{gathered}
f(\nu)=\frac{1}{2} \operatorname{Erfc}\left(\frac{\nu}{\sqrt{2}}\right)+\frac{1}{\sqrt{2 \pi}} e^{-\frac{\nu^{2}}{2}} \sum_{n=3}^{\infty} \frac{\left\langle x^{n}\right\rangle_{\mathrm{GC}}}{n !} H_{n-1}(\nu) . \\
\mathcal{L}(\nu)=\frac{1}{2 \sqrt{2} R_{0}} e^{-\frac{\nu^{2}}{2}}\left(1+\frac{1}{2 \sqrt{\pi}} \sum_{n=3}^{\infty} \sum_{i, j}^{i+2 j=n} \frac{(-1)^{j+1}}{i ! j !} \frac{\Gamma\left(j-\frac{1}{2}\right)}{\Gamma(j+1)}\left\langle x^{i} q^{2 j}\right\rangle_{\mathrm{G} \mathrm{C}} H_{i}(\nu)\right) .
\end{gathered}
$$

Euler characteristic density of the region above a threshold $x=\nu$ is a more interesting case. It is given by the average of the determinant of the Hessian matrix of the second derivatives of the field at the points where the first derivatives vanish Adler (1981), Longuet-Higgins (1957)

$$
\chi(\nu)=\int_{\nu}^{\infty} \mathrm{d} x \int \mathrm{d}^{3} x_{i j} P\left(x, x_{i}=0, x_{i j}\right) \operatorname{det}\left(x_{i j}\right) .
$$

It has been argued in Pogosyan, Gay \& Pichon (2009) that on $S_{2}$ the determinant should be that of the Hessian of the mixed covariant derivatives $\operatorname{det}\left(x_{; j}^{; i}\right)$. It is this choice that provides the density relative to the invariant volume element $R^{2} \sin ^{2} \theta \mathrm{d} \theta \mathrm{d} \phi$ and has a scalar trace equal to the Laplacian of the field. Using scaled invariant variables

$$
\operatorname{det}\left(x_{; j}^{; i}\right)=\frac{\sigma_{2}^{2}}{4}\left(J_{1}^{2}-(1-\beta) J_{2}\right) \equiv \sigma_{2}^{2} I_{2}
$$

where we have introduced another scaled quadratic invariant $I_{2}$. In terms of the eigenvalues of the Hessian, $\sigma_{2}^{2} I_{2}=\lambda_{1} \lambda_{2}$, while $\sigma_{2}^{2} J_{1}=\lambda_{1}+\lambda_{2}$ and $\sigma_{2}^{2}(1-\beta) J_{2}=\left(\lambda_{1}-\lambda_{2}\right)^{2}$.

In the Gaussian limit the Euler characteristic density becomes

$$
\chi(\nu)=\frac{\sigma_{2}^{2}}{8 \pi^{2} \sigma_{1}^{2}} \int_{-\infty}^{\infty} \mathrm{d} J_{1} \int_{0}^{\infty} \mathrm{d} J_{2} \int_{\frac{\nu+\gamma J_{1}}{\sqrt{1-\gamma^{2}}}}^{\infty} \mathrm{d} \zeta \exp \left[-\frac{1}{2} \zeta^{2}-\frac{1}{2} J_{1}^{2}-J_{2}\right]\left(J_{1}^{2}-(1-\beta) J_{2}\right)
$$

It evaluates to

$$
\chi(\nu)=\frac{\gamma^{2}}{4 \pi \sqrt{2 \pi} R_{*}^{2}} \nu e^{-\frac{\nu^{2}}{2}}+\frac{\beta}{8 \pi R_{*}^{2}} \operatorname{erfc}\left(\frac{\nu}{\sqrt{2}}\right)
$$

which differ from the well known Cartesian result by the $\beta \neq 0$ term. On a sphere which has a finite volume $4 \pi R^{2}$ it is appropriate to quote the total Euler characteristic in the whole volume, which, recalling the relation between $\gamma, \beta, R$ and $R_{*}$ becomes

$$
4 \pi R^{2} \chi(\nu)=\frac{R^{2}}{\sqrt{2 \pi} R_{0}^{2}} \nu e^{-\frac{\nu^{2}}{2}}+\operatorname{Erfc}\left(\frac{\nu}{\sqrt{2}}\right)
$$

which explicitly demonstrates that if $\nu=-\infty$. i.e the whole space is included in the excursion set, the total Euler characteristic is equal to that of a sphere, $4 \pi R^{2} \chi(-\infty)=2$, as expected.

Evaluation of the non-Gaussian expansion for $\chi(\nu)$ entails integration Eq. (2.3) with distribution function $P_{2 D}$ given by the Eq. (1.9). The procedure is similar to that in Cartesian space as elaborated in detail in Gay, Pichon \& Pogosyan (2012) and which led to the complete expression for the Euler characteristic to all orders first reported in Pogosyan, Gay \& Pichon (2009). Indeed, the quantity $I_{2}$ that we average over all the range of $J_{2}$ can be rewritten as $H_{2}\left(J_{1}\right)+\beta+(1-\beta) L_{1}\left(J_{2}\right)$. Thus only $l=0,1$ terms of the expansion, i.e containing $L_{0}\left(J_{2}\right)$ or $L_{1}\left(J_{2}\right)$, do not vanish after integration. Here we should limit ourselves with presenting only the result of the most practical use - up to 
the first, cubic in the field, non-Gaussian correction

$$
\begin{aligned}
& \chi(\nu)=\frac{\beta}{8 \pi R_{*}^{2}} \operatorname{Erfc}\left(\frac{\nu}{\sqrt{2}}\right)+\frac{1}{4 \pi \sqrt{2 \pi} R_{*}^{2}} \exp \left(-\frac{\nu^{2}}{2}\right) \\
& \times\left[\gamma^{2} H_{1}(\nu)+2 \gamma\left\langle q^{2} J_{1}\right\rangle+4\left\langle x I_{2}\right\rangle-\left(\gamma^{2}\left\langle x q^{2}\right\rangle+\gamma\left\langle x^{2} J_{1}\right\rangle\right) H_{2}(\nu)+\frac{\gamma^{2}}{6}\left\langle x^{3}\right\rangle H_{4}(\nu)\right. \\
& \left.\quad+\beta\left(-\left\langle x q^{2}\right\rangle H_{0}(\nu)+\frac{1}{6}\left\langle x^{3}\right\rangle H_{2}(\nu)\right)\right]
\end{aligned}
$$

where the Gram-Charlier moments of the non-primary variables $x$ and $I_{2}$ are understood as correspondent combinations of Gram-Charlier moments of the expansion variables $\zeta, J_{1}$ and $J_{2}$. The first term of Eq. (2.8) is the Gaussian result on the sphere that is responsible for the total Euler number of the excursion set to be that of the total sphere when $\nu=-\infty$. The last $\propto \beta$ terms is a correction to non-Gaussian result due to the curvature of the sphere. In conclusion we as well write explicitly the result for the total Euler number above threshold $\nu$

$$
\begin{aligned}
& 4 \pi R^{2} \chi(\nu)=\operatorname{Erfc}\left(\frac{\nu}{\sqrt{2}}\right)+\frac{2}{\sqrt{2 \pi}} \exp \left(-\frac{\nu^{2}}{2}\right)\left(\frac{1}{6}\left\langle x^{3}\right\rangle H_{2}(\nu)-\left\langle x q^{2}\right\rangle H_{0}(\nu)\right) \\
& +\frac{R^{2}}{\sqrt{2 \pi} R_{0}^{2}} \exp \left(-\frac{\nu^{2}}{2}\right) \\
& \quad \times\left[H_{1}(\nu)+\frac{2}{\gamma}\left\langle q^{2} J_{1}\right\rangle+\frac{4}{\gamma^{2}}\left\langle x I_{2}\right\rangle-\left(\left\langle x q^{2}\right\rangle+\frac{1}{\gamma}\left\langle x^{2} J_{1}\right\rangle\right) H_{2}(\nu)+\frac{1}{6}\left\langle x^{3}\right\rangle H_{4}(\nu)\right] .
\end{aligned}
$$

\section{Extrema counts on $S_{2}$ beyond the Gaussian limit}

The number density of extrema above a threshold $\nu$ is given by an integral very similar to the Euler characteristic Adler (1981), Longuet-Higgins (1957)

$$
n_{\text {ext }}(\nu)=\int_{\nu}^{\infty} d x \int \mathrm{d}^{3} x_{i j} P\left(x, x_{i}=0, x_{i j}\right)\left|x_{i j}\right| \Theta_{e x t}\left(\lambda_{m}\right) .
$$

where the theta function $\Theta_{\text {ext }}\left(\lambda_{m}\right)$ chooses the regions of integration in the space of second derivatives with appropriate to the particular extremum type signs of the Hessian eigenvalues. In 2D, assuming $\lambda_{1} \geqslant \lambda_{2}, \Theta_{\text {ext }}\left(\lambda_{m}\right)=\Theta\left(-\lambda_{1}\right)$ for maxima, $=\theta\left(\lambda_{2}\right)$ for minima, and $=\theta\left(\lambda_{1}\right) \theta\left(-\lambda_{2}\right)$ for saddle points. Indeed, we have a well-known topological relation

$$
\chi(\nu)=n_{\max }(\nu)-n_{\mathrm{sad}}(\nu)+n_{\min }(\nu)
$$

The integral Eq. (3.1) has a very transparent form when the Hessian is described in invariant variables. It is equivalent to Eq. (2.5), except that the limits of integration over $J_{1}$ are partitioned into the regions of the fixed sign of the determinant $I_{2}$. Namely, maxima correspond to the range $J_{1} \in\left(-\infty,-\sqrt{(1-\beta) J_{2}}\right)$, minima to $J_{1} \in\left(\sqrt{(1-\beta) J_{2}}, \infty\right)$ and saddle points to $J_{1} \in\left(-\sqrt{(1-\beta) J_{2}}, \sqrt{(1-\beta) J_{2}}\right)$.

Calculations for differential density of extremal points, $\partial n_{\text {ext }} / \partial(\nu)$ can be carried out analytically even for the general expression Eq, (1.9) (see discussion in Gay, Pichon \& Pogosyan (2012) for the flat case). The resulting expressions are cumbersome, and here we limit ourselves to presenting results for the total density of extrema in the first nonGaussian order only. The total number density of maxima is given by

$$
n_{\max }=\frac{\sigma_{2}^{2}}{4 \sigma_{1}^{2}} \int_{0}^{\infty} \mathrm{d} J_{2} \int_{-\infty}^{-\sqrt{(1-\beta) J_{2}}} \mathrm{~d} J_{1} P_{2 D}\left(q^{2}=0, J_{1}, J_{2}\right)\left|J_{1}^{2}-(1-\beta) J_{2}\right|
$$


and, similarly, for the minima and the saddle points. The result is

$$
\begin{aligned}
n_{\max / \min } & =\frac{(1-\beta)^{3 / 2}+\beta \sqrt{3-\beta}}{8 \pi \sqrt{3-\beta} R_{*}{ }^{2}} \pm \frac{6(3-\beta)\left\langle q^{2} J_{1}\right\rangle-(5-3 \beta)\left\langle J_{1}^{3}\right\rangle+6(1-\beta)\left\langle J_{1} J_{2}\right\rangle}{6 \pi \sqrt{2 \pi} R_{*}{ }^{2}(3-\beta)^{2}}, \\
n_{\mathrm{sad}} & =\frac{(1-\beta)^{3 / 2}}{4 \pi \sqrt{3-\beta} R_{*}{ }^{2}},
\end{aligned}
$$

where we immediately see that $n_{\max }+n_{\min }-n_{\text {sad }}=\beta /\left(4 \pi R_{*}^{2}\right)=2 /\left(4 \pi R^{2}\right)$ as expected. The total number of saddles, as well as of all the extremal points, $n_{\max }+n_{\min }+n_{\text {sad }}$, are preserved in the first order (the latter following from the former), but the symmetry between the minima and the maxima is broken.

It is instructive to look how the Gaussian extrema counts are modified by the properties of spherical space when the curvature radius is large relative to the typical extrema separation scale $R_{*}$, i.e when $\beta$ is small. Up to the first order in $\beta$

$$
\begin{aligned}
n_{\max / \min } & \sim \frac{1}{8 \sqrt{3} \pi R_{*}^{2}}+\frac{9-4 \sqrt{3}}{36 \pi R^{2}} \approx \frac{1}{8 \sqrt{3} \pi R_{*}^{2}}\left(1+0.8 R_{*}^{2} / R^{2}\right) \\
n_{\mathrm{sad}} & \sim \frac{1}{4 \sqrt{3} \pi R_{*}^{2}}-\frac{2}{3 \sqrt{3} \pi R^{2}} \approx \frac{1}{4 \sqrt{3} \pi R_{*}^{2}}\left(1-2.7 R_{*}^{2} / R^{2}\right)
\end{aligned}
$$

This shows that being on a sphere, increases the number density of maxima and minima, but decrease (and in more significant way) the number of saddles. Incidently, assuming large-angle CMB power spectrum, truncated at $l=30$ gives $\beta \approx 1 / 170$, i.e $1 \%$ correction to the count of extrema relative to the flat-sky approximation.

\section{References}

Adler, R. J. The Geometry of Random Fields. The Geometry of Random Fields, Chichester: Wiley, 1981.

Bardeen, J. M., Bond, J. R., Kaiser, N., \& Szalay, A. S. The statistics of peaks of Gaussian random fields. ApJ, 304:15-61, May 1986.

Codis, S., Pichon, C., Pogosyan, D., Bernardeau, F., \& Matsubara, T. Non-Gaussian Minkowski functionals and extrema counts in redshift space. MNRAS, 435:531-564, October 2013.

Doroshkevich, A. G. The space structure of perturbations and the origin of rotation of galaxies in the theory of fluctuation. Astrofizika, 6:581-600, 1970.

Gay, C., Pichon, C., \& Pogosyan, D. Non-Gaussian statistics of critical sets in 2D and 3D: Peaks, voids, saddles, genus, and skeleton. Phys. Rev. D, 85(2):023011, January 2012.

Longuet-Higgins, M. S. The statistical analysis of a random, moving surface. Royal Society of London Philosophical Transactions Series A, 249:321-387, February 1957.

Marcos-Caballero, A., Fernández-Cobos, R., Martínez-González, E., \& Vielva, P. The shape of CMB temperature and polarization peaks on the sphere. JCAP, 4:058, April 2016.

Matsubara, T. Statistics of Smoothed Cosmic Fields in Perturbation Theory. I. Formulation and Useful Formulae in Second-Order Perturbation Theory. ApJ, 584:1-33, February 2003.

Matsubara, T. Analytic Minkowski functionals of the cosmic microwave background: Secondorder non-Gaussianity with bispectrum and trispectrum. Phys. Rev. D, 81(8):083505, April 2010.

Pogosyan, D., Gay, C., \& Pichon, C. Invariant joint distribution of a stationary random field and its derivatives: Euler characteristic and critical point counts in 2 and 3D. Phys. Rev. $D, 80(8): 081301$, October 2009.

Pogosyan, D., Gay, C., \& Pichon, C. Erratum: Invariant joint distribution of a stationary random field and its derivatives: Euler characteristic and critical point counts in 2 and 3D [Phys. Rev. D 80, 081301 (2009)]. Phys. Rev. D, 81(12):129901, June 2010.

Pogosyan, D., Pichon, C., \& Gay, C. Non-Gaussian extrema counts for CMB maps. Phys. Rev. $D, 84(8): 083510$, October 2011. 